\title{
Exploring the inside details of virions by electron microscopy
}

\author{
Zheng Liu ${ }^{1}$, Jingqiang Zhang ${ }^{2 \bowtie}$ \\ 1 Department of Biochemistry and Molecular Biophysics, Columbia University, New York, NY 10032, USA \\ 2 College of Life Science, SunYat-sen University, Guangzhou 510275, China
}

Received: 11 January 2016 / Accepted: 24 January 2016 / Published online: 22 April 2016

Spherical virions can be structurally divided into two compositions: the outer protein shell, and its content including the viral genome and enzymes. Threedimensional virus structures are the key to understanding the virus life cycles and their mechanisms for infecting hosts, which can contribute to anti-viral drug design. These structures can be solved either by X-ray crystallization (Harrison et al. 1978) or by cryo-electron microscopy (cryo-EM) (Dubochet et al. 1988), or by combining both methods (Guu et al. 2009). In retrospect, the X-ray method had begun to solve viral structures at high resolution with atomic information since the late 1970s (Harrison et al. 1978; AbadZapatero et al. 1980), while cryo-EM, considered as a blobology method, only provided low- or mediumresolution information from the 1980s (Dubochet et al. 1988). In recent years, cryo-EM has emerged to rival with X-ray on the high-resolution structure determination of spherical viruses (Grant and Grigorieff 2015; Liu et al. 2016). In spite of a wealth of virus structures determined by these methods, the solved parts were limited to the viral capsid, while the remaining moieties, like the viral genomes and virus-self-functional enzymes within, were rarely visualized. Strikingly, in a recent paper published in Science (Liu and Cheng 2015), Drs. Liu and Cheng managed to discern the full genome and polymerase of cytoplasmic polyhedrosis virus (CPV), using their new approach adapted from traditional single-particle analysis of icosahedral viruses.

The CPV belongs to the Reoviridae family, whose members cause diverse diseases in their hosts ranging from plants, insects, to humans. CPVs cause diseases only in arthropods and therefore are considered as potential biological insecticides to combat their hosts

$\triangle$ Correspondence: lsszhjq@sysu.edu.cn (J. Zhang) injuries to agriculture. The Bombyxmori CPV (BmCPV), the exact species used in the presented study, can cause serious flaccidness in silkworm, posing a big burden in sericulture development in East Asia. The genome of $\mathrm{BmCPV}$ is composed of 10 segments of double-stranded (ds) RNA. The naturally mature virions encapsulate their own RNA-dependent RNA polymerases (RdRps) into the capsid and transcribe the minus-strand RNA to mRNA inside the intact capsid, which usually occurs in host cell's cytoplasm. In comparisons with other members of Reoviridae having a $T=13$ layer (here $T$ is the triangulation number), $\mathrm{CPV}$ virion only has a single layer capsid, adopting $T=1$ lattice, with $\sim 70 \mathrm{~nm}$ in diameter (Fig. 1A). Purification of virus from the silkworm midgut usually yields two kinds of particles-empty capsids and complete virions. Treated with GTP properly, the virions performing transcription (transcribing CPV, TCPV) can also be obtained from their nontranscribing state (non-transcribing CPV, NCPV).

To reveal the structure of CPV, both X-ray and singleparticle cryo-EM were applied. Probably due to the intrinsic flexibility of some viral components, CPV had eluded the efforts of the X-ray method, while cryo-EM advanced the structural studies while steadily improving resolutions (Hill et al. 1999; Zhang et al. 1999; Xia et al. 2003; Zhou et al. 2003). As experience with this virus was accumulated, CPV was considered the benchmark sample for high-resolution study of singleparticle cryo-EM. In 2008, Yu et al. reported the first high-resolution structure of BmCPV at $3.88 \AA$ (Fig. 1B). More high-resolution structure of CPV then followed, including a 3.1-Å structure and a 3.9-Å structure of the more complete CPV (Cheng et al. 2011; Yu et al. 2011), and a cryo-EM structure of the transcribing CPV (Yang et al. 2012). However, the internal structures of genome and RdRps have been kept in the dark until Liu and Cheng's work. 

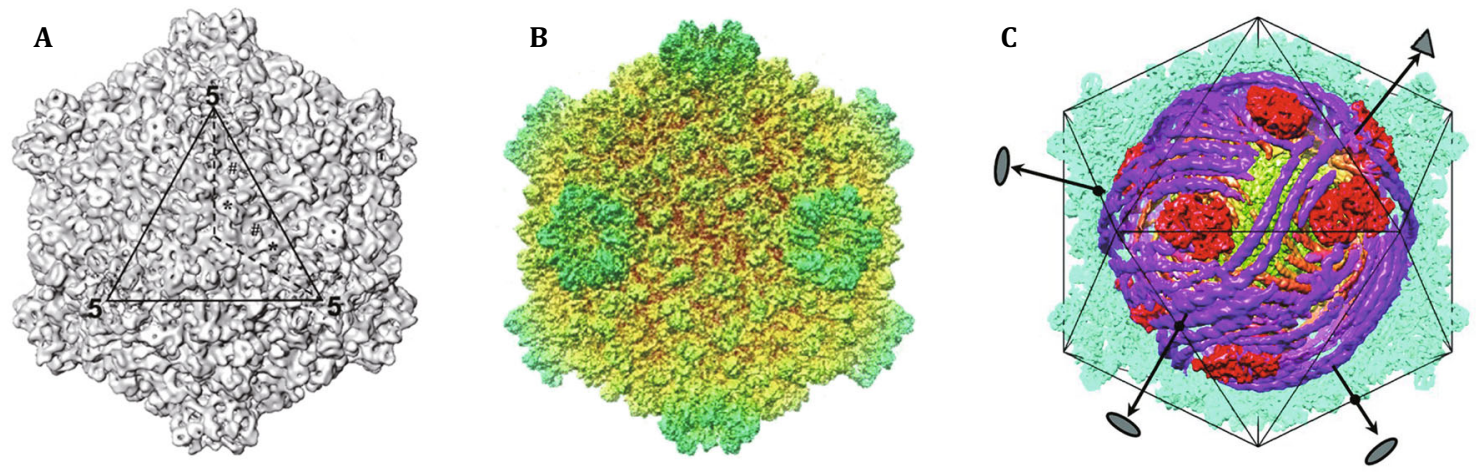

Fig. 1 Cryo-EM of CPV. A A low-resolution structure of CPV (Zhang et al. 1999). B The 3.88- $\AA$ CPV structure, the first high-resolution macromolecule structure determined by single-particle cryo-EM (Yu et al. 2008). C The CPV structure reconstructed by symmetrymismatch reconstruction (Liu and Cheng 2015)

Aiming to build the structure of the genome and RdRps of CPV, the authors developed a so-called symmetry-mismatch reconstruction in their study. At the heart of the method, it finds out the accurate positional parameters for particles containing the genome and enzymes as a normal single-particle analysis dose (Frank 2009). The study took a two-step process to determine the parameters: first, making use of the icosahedral symmetry of the outer capsid shell, virions orientation searching was only limited to one asymmetric unit of the icosahedral space; second, to get the real azimuth of the genomes in each particle, here the first-step generated orientation was relaxed to full spherical space by allocating 60 possibilities, and then refined by iteratively aligning the interested inner part of each raw images with the references. The initial reference was obtained by masking the shell from the full virus map reconstructed using raw images with the random selection of one of the 60 equivalent orientations. During the second step, in order to eliminate the interference from the capsid and to reliably determine the azimuthal parameters, the authors used the capsid structure projection according to the particle orientation to mask the protein shell on the raw image by use of pixel subtraction, with the consideration of CTF correction and grayscale adjustment. The final structure was reconstructed from raw virus images other than the genome-extracted images using the determined positional parameters.

With their new strategy, Liu and Cheng solved the structures of both the NCPV and TCPV in the presented paper. The arrangements of genome and RdRps are alike in both virions, while the interactions between genomic RNA and RdRps differ in the two states. Their genome and RdRps adopts a pseudo-D3 symmetric organization, by which further reconstruction enhanced the resolutions to allow building a $\mathrm{C} \alpha$ model. Some extra densities are seen in the active sites in NCPV and TCPV and nucleotides can be assigned into them. From both maps, a fragment of dsRNA is observed to bind to each of the 12 RdRps bracelets, which shifts in NCPV compared to that in TCPV, which is interpreted as virion transcription at initiation stage. The channels for the exit of transcript and RNA template are partially blocked in NCPV RdRps, but completely open in TCPV RdRps. A loop in RdRps adopts a retracted conformation in NCPV and becomes extended in TCPV, thus it is interpreted as the switch loop. These details about the viral functional enzymes and genome are not seen in previous virus structure studies, which have been going on for nearly four decades (Harrison et al. 1978). The current work is therefore a landmark in structural virology.

On the basis of the internal structures of NCPV and TCPV plus previous knowledge on the Reoviridae viruses, Liu and Cheng proposed a dsRNA virus replication and transcription model, although structures of some potential states were still lacking in this study. According to their model, the replication initiates from the plus-strand RNA, which passes through the template entry channel of RdRps. The resulting dsRNA then exits at the template channel exit and binds to the bracelet domain of RdRps to prepare for transcription. Upon transcription, dsRNA disassociation from the binding site triggers the opening of the transcript and template exit channels, as well as the extension of the switch loop. The dsRNA is dissembled at the entrance of the template entry channel likely catalyzed by RdRps, and then the minus strand enters into the channel serving as template for synthesizing mRNA, while the plus strand stays outside the channel by binding its $5^{\prime}$ cap to the RdRps. When the finished transcript exits the transcript channel, the template associates with the original plusstrand RNA to form dsRNA for next round transcription. 
This four-channel coordinated model for dsRNA virus replication and transcription is a principle finding of Liu and Cheng's structure analysis.

Regarding the methods, as the authors mentioned, similar idea had been used elsewhere including solving the bacteriophages local symmetry mismatched details (Tao et al. 1998; Morais et al. 2001; Jiang et al. 2006), and the kelp fly virus vertex (Briggs et al. 2005). A further usage of asymmetrical reconstruction named focused asymmetrical reconstruction was to reveal the tandem symmetry mismatches in T7 phage (Guo et al. 2013). However, neither did reach high resolution for these studies, nor were the genomes of these viruses solved. The authors also hinted that the methods used in their study are well suited to other macromolecules with mixed symmetry.

The current work's data were collected on a CCD camera instead of a superior direct detector, which contributes a lot to image quality improvement and image processing (Liu and Zhang 2014). Thus, the resolutions of the NCPV and TCPV were not sufficient to allow the authors to build an accurate atomic model. Nevertheless, the work surely demonstrated that the genome is solvable by averaging a large amount of particles. The authors also admitted that the D3 symmetry in the dsRNA organization probably did not reflect the true arrangement given the fact that the RNA genome differs in size among the 10 segments in each virus.

Further application of direct detectors for data collection, combining with other image-processing algorithms, and improved specimen-preparation methods should bring other advances in the use of cryo-EM for structural virology. With the outstanding success of the current study, the gate is open for structural studies of internal contents of other viruses like phages and other infectious viruses (Dai et al. 2013; Guo et al. 2014; Serwer et al. 2014; Manokaran et al. 2015). Because viruses represent the simplest life-being and are the direct targets for anti-viral drugs, the findings may even unveil more fundamental mechanisms related to current biology and herald the dawn of single-particle cryoEM as a method to facilitate rational anti-viral drug design.

Acknowledgments Authors thank Dr. Cui Jinmin and Cristina Gutierrez-Vargas for reading through the manuscript and for their suggestions on the English revising. This work was supported by a grant from the National Natural Science Foundation of China (31170691 to J.Z.).

Open Access This article is distributed under the terms of the Creative Commons Attribution 4.0 International License (http:// creativecommons.org/licenses/by/4.0/), which permits unrestricted use, distribution, and reproduction in any medium, provided you give appropriate credit to the original author(s) and the source, provide a link to the Creative Commons license, and indicate if changes were made.

\section{References}

Abad-Zapatero C, Abdel-Meguid SS, Johnson JE, Leslie AG, Rayment I, Rossmann MG, Suck D, Tsukihara T (1980) Structure of southern bean mosaic virus at $2.8 \AA$ resolution. Nature 286:33-39

Briggs JAG, Huiskonen JT, Fernando KV, Gilbert RJC, Scotti P, Butcher SJ, Fuller SD (2005) Classification and three-dimensional reconstruction of unevenly distributed or symmetry mismatched features of icosahedral particles. J Struct Biol 150: 332-339

Cheng L, Sun J, Zhang K, Mou Z, Huang X, Ji G, Sun F, Zhang J, Zhu P (2011) Atomic model of a cypovirus built from cryo-EM structure provides insight into the mechanism of mRNA capping. Proc Natl Acad Sci USA 108:1373-1378

Dai HS, Liu Z, Jiang W, Kuhn RJ (2013) Directed evolution of a virus exclusively utilizing human epidermal growth factor receptor as the entry receptor. J Virol 87:11231-11243

Dubochet J, Adrian M, Chang JJ, Homo JC, Lepault J, McDowall AW, Schultz P (1988) Cryo-electron microscopy of vitrified specimens. Q Rev Biophys 21:129-228

Frank J (2009) Single-particle reconstruction of biological macromolecules in electron microscopy-30 years. Q Rev Biophys 42:139-158

Grant T, Grigorieff N (2015) Measuring the optimal exposure for single particle cryo-EM using a $2.6 \AA$ reconstruction of rotavirus VP6. eLife 4:e06980

Guo F, Liu Z, Vago F, Ren Y, Wu W, Wright ET, Serwer P, Jiang W (2013) Visualization of uncorrelated, tandem symmetry mismatches in the internal genome packaging apparatus of bacteriophage T7. Proc Natl Acad Sci USA 110:6811-6816

Guo F, Liu Z, Fang PA, Zhang Q Wright ET, Wu W, Zhang C, Vago F, Ren Y, Jakana J, Chiu W, Serwer P, Jiang W (2014) Capsid expansion mechanism of bacteriophage $\mathrm{T} 7$ revealed by multistate atomic models derived from cryo-EM reconstructions. Proc Natl Acad Sci USA 111:E4606-E4614

Guu TSY, Liu Z, Ye QZ, Mata DA, Li KP, Yin CC, Zhang JQ, Tao YJ (2009) Structure of the hepatitis E virus-like particle suggests mechanisms for virus assembly and receptor binding. Proc Natl Acad Sci USA 106:12992-12997

Harrison SC, Olson AJ, Schutt CE, Winkler FK, Bricogne G (1978) Tomato bushy stunt virus at $2.9 \AA$ resolution. Nature 276:368-373

Hill CL, Booth TF, Prasad BV, Grimes JM, Mertens PP, Sutton GC, Stuart DI (1999) The structure of a cypovirus and the functional organization of dsRNA viruses. Nat Struct Biol 6:565-568

Jiang W, Chang J, Jakana J, Weigele P, King J, Chiu W (2006) Structure of epsilon 15 bacteriophage reveals genome organization and DNA packaging/injection apparatus. Nature 439:612-616

Liu H, Cheng L (2015) Cryo-EM shows the polymerase structures and a nonspooled genome within a dsRNA virus. Science 349:1347-1350

Liu Z, Zhang J-J (2014) Revolutionary breakthrough of structure determination-recent advances of electron direct detection device application in cryo-EM. Acta Biophys Sin 30:12

Liu Z, Guo F, Wang F, Li TC, Jiang W (2016) 2.9 Å resolution cryoEM 3D reconstruction of close-packed virus particles. Structure 24:319-328 
Manokaran G, Finol E, Wang CL, Gunaratne J, Bahl J, Ong EZ, Tan HC, Sessions OM, Ward AM, Gubler DJ, Harris E, Garcia-Blanco MA, Ooi EE (2015) Dengue subgenomic RNA binds TRIM25 to inhibit interferon expression for epidemiological fitness. Science 350:217-221

Morais MC, Tao YZ, Olson NH, Grimes S, Jardine PJ, Anderson DL, Baker TS, Rossmann MG (2001) Cryoelectron-microscopy image reconstruction of symmetry mismatches in bacteriophage phi 29. J Struct Biol 135:38-46

Serwer P, Wright ET, Liu Z, Jiang W (2014) Length quantization of DNA partially expelled from heads of a bacteriophage T3 mutant. Virology 456:157-170

Tao YZ, Olson NH, Xu W, Anderson DL, Rossmann MG, Baker TS (1998) Assembly of a tailed bacterial virus and its genome release studied in three dimensions. Cell 95:431-437

Xia Q Jakana J, Zhang JQ, Zhou ZH (2003) Structural comparisons of empty and full cytoplasmic polyhedrosis virus. ProteinRNA interactions and implications for endogenous RNA transcription mechanism. J Biol Chem 278:1094-1100
Yang C, Ji G, Liu H, Zhang K, Liu G, Sun F, Zhu P, Cheng L (2012) Cryo-EM structure of a transcribing cypovirus. Proc Natl Acad Sci USA 109:6118-6123

Yu X, Jin L, Zhou ZH (2008) $3.88 \AA$ structure of cytoplasmic polyhedrosis virus by cryo-electron microscopy. Nature 453:415-419

Yu XK, Ge P, Jiang JS, Atanasov I, Zhou ZH (2011) Atomic model of CPV reveals the mechanism used by this single-shelled virus to economically carry out functions conserved in multishelled reoviruses. Structure 19:652-661

Zhang H, Zhang J, Yu X, Lu X, Zhang Q Jakana J, Chen DH, Zhang X, Zhou ZH (1999) Visualization of protein-RNA interactions in cytoplasmic polyhedrosis virus. J Virol 73:1624-1629

Zhou ZH, Zhang H, Jakana J, Lu XY, Zhang JQ (2003) Cytoplasmic polyhedrosis virus structure at $8 \AA$ by electron cryomicroscopy: structural basis of capsid stability and mRNA processing regulation. Structure 11:651-663 